\title{
Possible Beneficial Effect of Exercise, by Reducing Oxidative Stress, on the Incidence of Preeclampsia
}

\author{
SEONAE YEO, R.N., Ph.D., ${ }^{1}$ and SANDRA T. DAVIDGE, Ph.D. ${ }^{2}$
}

\begin{abstract}
We hypothesize that regular exercise enhances antioxidative enzymes in pregnant women, which reduce oxidative stress and, thus, the incidence of preeclampsia. Oxidative stress with enhanced lipid peroxide formation could lead to endothelial dysfunction in preeclampsia. Other conditions, such as increased transferrin saturation and decreased iron-binding capacity, directly and indirectly promote the process of oxidative stress and subsequent endothelial dysfunction. Exercise increases oxidative metabolism and produces a prooxidant environment. This acidic environment during exercise (at or beyond anaerobic threshold) promotes oxygen release from hemoglobin and increases in $\mathrm{Po}_{2}$ in tissues, as well as releases iron from transferrin. When exercise is repeated regularly, the body promptly adjusts so that oxidative stress is eliminated or reduced. The body's adaptations to a regular exercise habit seem to have an antioxidant effect. In humans, training effects have been identified with an enhanced activity of antioxidative enzymes. Another concerted adaptation that regular exercise brings to women's bodies is resistance against production of prooxidants by increasing the number of mitochondria. Equally important is a training effect that decreases susceptibility to lipid peroxidation. Evidence suggests that physically active women are less likely to develop preeclampsia. In theory, intracellular and extracellular conditions resulting from regular exercise should counteract the enhancement of oxidative stress, thus interfering with the process leading to endothelial dysfunction. This position paper describes a hypothesis and includes a brief review of exercise physiology and biochemical research in preeclampsia. Unlike other preventive treatments, such as aspirin or calcium supplements, a regular exercise habit leads to a positive and healthy lifestyle without concern of side effects.
\end{abstract}

\section{INTRODUCTION}

$\mathbf{P}$ REECLAMPSIA IS A COMMON DISORDER of human pregnancy in which the normal hemodynamic response to pregnancy is compromised. It remains a leading cause of maternal morbidity ( $5 \%-7 \%$ of all pregnancies) and perinatal mortal- ity. ${ }^{1-3}$ Preeclampsia is diagnosed primarily by the onset of hypertension and proteinuria in the latter half of gestation. Other manifestations of preeclampsia include generalized vasoconstriction, increased vasoactivity, reduced perfusion to organs, and platelet activation. 4,5 Currently, there are no conclusive preventive treatments available

${ }^{1}$ The University of Michigan, School of Nursing Division of Health Promotion and Risk Reduction, Ann Arbor, Michigan.

${ }^{2}$ Perinatal Research Centre, University of Alberta, Alberta, Canada.

This work is partially supported by the University of Michigan Office of Vice President for Research.

S.T.D. is a Senior Scholar of the Alberta Heritage Foundation for Medical Research (AHFMR) and Canadian Institutes of Health Research. 
because both the etiology and pathophysiology of preeclampsia are poorly understood. An emerging theory explaining this mysterious and ominous disease involves oxidative stress. ${ }^{6}$ Juxtaposed to this theory is the role of oxidative stress in acute (i.e., single bout) and regular exercises. ${ }^{7}$ Effects of acute vs. regular exercise are opposing, and there is evidence to suggest that physically active women are less likely to develop preeclampsia. ${ }^{8}$ This paper describes a brief summary of the pathology of preeclampsia and the physiology of exercise, focusing specifically on the role of oxidative stress.

\section{OXIDATIVE STRESS}

Oxidative stress is a general term used to describe a state of damage caused by reactive oxygen species. ${ }^{9}$ This damage can affect a specific molecule or the entire organism. The level of oxidative stress is determined by the balance between the rate at which prooxidant is produced and the rate at which it is removed by an antioxidative mechanism. Thus, oxidative stress is an imbalance between prooxidant and antioxidants resulting in an increase in prooxidants. Prooxidants are important in the commencement of endothelial cell dysfunction in many vascular diseases, including atherosclerosis, hypertension, diabetes, and preeclampsia. Prooxidants include free radicals, such as superoxide anions and hydroxyl radicals, as well as other reactive oxygen species, such as hydrogen peroxide $\left(\mathrm{H}_{2} \mathrm{O}_{2}\right)$. Oxygen free radicals are produced from a number of cellular sources, including mitochondrial electron transport activity, NADPH oxidase, arachidonate metabolism, and nitric oxide synthase (particularly if the intracellular concentrations of L-arginine are low). ${ }^{6}$ In addition, factors in the circulation (such as xanthine oxidase and neutrophils) can produce oxygen free radicals that will react with endothelial cells. ${ }^{6}$ The prooxidant environment is widespread for endothelial cells, as these cells not only produce their own free radicals but also are constantly exposed to factors from the circulation.

When polyunsaturated fatty acids in the cell membrane of living organisms are attacked by free radicals in the presence of molecular oxygen, a chain of chemical reactions can occur, eventually leading to the disintegration of fatty acid and formation of hydrocarbon gases (e.g., pentane) and aldehydes (e.g., malondialdehyde). This process is called lipid peroxidation. Peroxidation of lipids exposed to oxygen is responsible not only for deterioration of foods but also for damage to tissues in humans, where it may play a role in, for example, cancer, inflammatory disease, and aging. ${ }^{10}$ Lipid peroxidation is a chain reaction providing a continuous supply of free radicals that initiate further peroxidation. The level of serum lipid peroxide is believed to indicate the body's overall oxidative stress.

The rate at which damage is produced also is determined by how fast reactive oxygen species are created and then inactivated by molecules called antioxidants. To prevent the free radical propagation effect, the body uses antioxidants to stop the biochemical chain reaction. Antioxidants are compounds and reactions that dispose of reactive oxygen species by scavenging them, suppressing their formation, or opposing their actions. Antioxidant mechanisms are complex and multifactorial. Antioxidants include the enzymes glutathione peroxidase, superoxide dismutase, and catalase. These enzymes derive sequences of reactions that can quickly convert oxygen radicals into harmless water. Other categories of antioxidants include the water-soluble and lipidsoluble antioxidants, such as ascorbic acid and vitamin $\mathrm{E}$, respectively. These vitamins react with free radicals in ways that convert the vitamins into inactive compounds that leave the body, thereby depleting tissues of the vitamins. In a normal state, there is an appropriate prooxidant-antioxidant balance, but this balance is altered when production of prooxidants is increased greatly or when levels of antioxidants are diminished. ${ }^{11}$

\section{ROLE OF OXIDATIVE STRESS IN WOMEN WITH PREECLAMPSIA}

Currently, there are many hypotheses that are the subject of extensive investigation because the etiology of preeclampsia is still unknown. Some conditions that have been hypothesized as leading to preeclampsia include placental ischemia, immune maladaptation, genetic imprinting, and very low density lipoprotein (VLDL) vs. toxicitypreventing activity, including oxidative stress. ${ }^{12}$ Ultimately, the predominant pathophysiological feature of preeclampsia is likely due to vascular endothelial cell dysfunction, as evidenced by the presence of glomerular endotheliosis as well as 


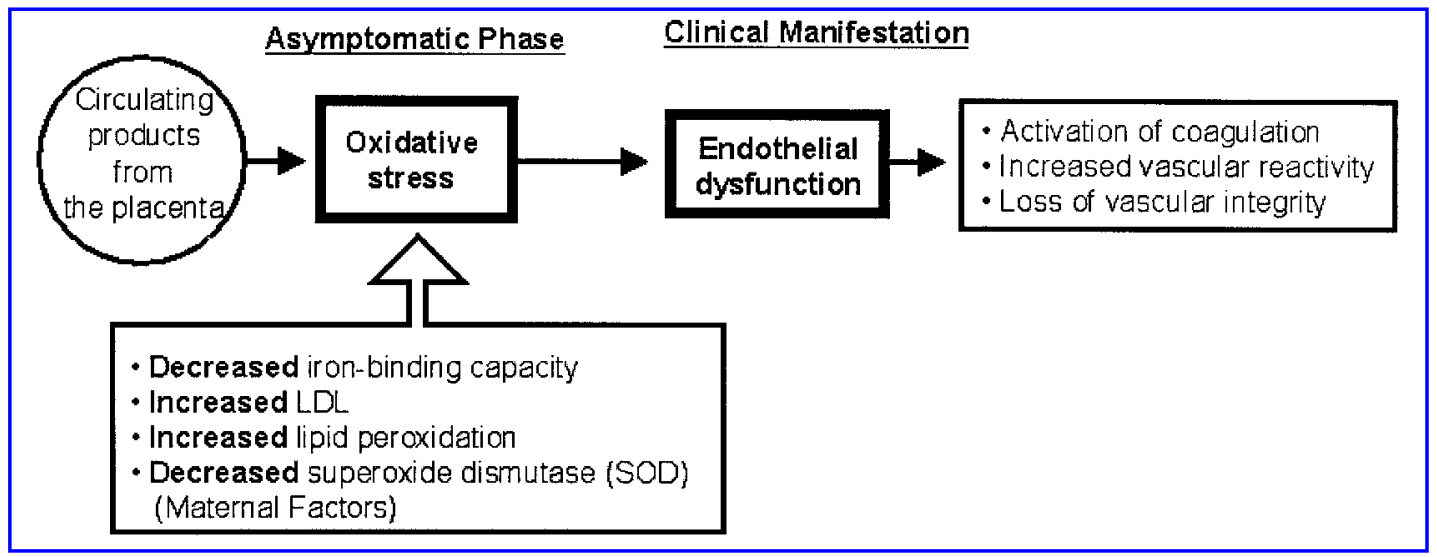

FIG. 1. Development of preeclampsia and the role of oxidative stress.

biochemical assessment of circulating products released from dysfunctional endothelial cells (such as cellular fibronectin, factor VIII antigen, and endothelin). In vitro studies of cultured endothelial cells and isolated arteries also indicate that endothelial cell dysfunction contributes to the pathophysiology of preeclampsia. ${ }^{6}$ One prevailing hypothesis is that oxidative stress is a mediator of endothelial cell dysfunction in preeclampsia.

Interestingly, normal pregnancy may be a state of mild oxidative stress, as serum lipid peroxides appear to increase in normal pregnant women compared with nonpregnant women. ${ }^{13-15}$ However, lipid peroxide levels are further increased in preeclampsia, ${ }^{10,12,16,17}$ whereas healthy pregnant women produce abundant antioxidants to remove prooxidants promptly. In addition, circulating free fatty acids, which can lead to the formation of lipid peroxides, are already increased 15-20 weeks before the onset of clinical disease. ${ }^{18}$ Indeed, oxidative stress leading to enhanced lipid peroxide formation could cause endothelial dysfunction in preeclampsia, ${ }^{6,19-25}$ which gives rise to the activation of the coagulation cascade, increased vascular reactivity, and loss of vascular integrity. Lipid peroxides stimulate prostaglandin $\mathrm{H}$ synthase but inhibit prostacyclin synthase. This may provide a direct link between increased lipid peroxide levels in preeclampsia and decreased synthesis of the vasorelaxant prostacyclin. ${ }^{17,26}$ Moreover, the oxygen-derived free radical, superoxide anion, can react with nitric oxide, producing peroxynitrite, which subsequently could reduce bioavailability of the potent vasodilator, nitric oxide. Evidence of elevated peroxynitrite formation has been described in both placental ${ }^{27}$ and maternal blood vessels of women with preeclampsia. ${ }^{22}$

There is other evidence that indicates enhanced oxidative stress among women with preeclampsia (Fig. 1). For example, increased transferrin saturation and decreased unsaturated iron-binding capacity in preeclampsia may occur subsequent to oxidative stress and then further promote oxidative stress by decreasing serum antioxidant buffering against redox-active iron. ${ }^{28}$ Highly toxic radicals, such as hydroxyl radical, initiate lipid peroxidation and reactions causing propagation of lipid peroxidation. Generation of these radicals requires the presence of a low molecular weight pool of iron or other transition metals. Thus, this condition directly and indirectly promotes the process of oxidative stress and could subsequently lead to endothelial dysfunction.

\section{EXERCISE AND OXIDATIVE STRESS}

Single-bout exercise increases oxidative metabolism, and this produces a prooxidant environment. ${ }^{29}$ In contrast, when exercise is repeated, the body becomes adjusted to this stress in such a way that it can react promptly so that the condition of oxidative stress is eliminated or reduced before it causes damage to cellular structure. Thus, the adaptations to a regular exercise habit seem to have an antioxidant protective effect. 7,30 Although these hypotheses have been tested with animal models, the applicability of the results remained untested because relatively few studies have been conducted with human models. In determining the relationship between regular exercise and antioxidant protective effects, psycho- 
logical and behavioral aspects of measurements make human models complex. In the following section, mechanisms of a single bout of exercise (prooxidant effect) and regular exercise (antioxidant effect) are briefly discussed.

\section{Single bout of exercise}

Oxidative stress caused by a single bout of exercise demonstrates a dose-response and threshold effect. ${ }^{31}$ Exercise-induced lipid peroxidation in human skeletal muscle was proportional to the intensity of the exercise. The same study also reported that this lipid peroxidation was initiated at the level of the anaerobic threshold. ${ }^{31}$ Oxidative stress-modulating factors, that is, factors that increase prooxidants, caused by single bouts of exercise are increased by electron transport chain activity, local inflammation, increased xanthine oxide, and depletion of antioxidants. ${ }^{32}$

During aerobic exercise, whole body oxygen consumption can increase up to 10 -fold. ${ }^{11}$ Exercise increases respiration, with increased flow of electrons in the electron transport chain, and this elevates the level of prooxidants. This happens because instead of electrons being passed to cytochrome oxidase, oxygen is reduced by one electron. ${ }^{29}$ Exhaustion of ATP pools leads to high intercellular levels of ADP. This condition triggers the catabolism of ADP and conversion of xanthine dehydrogenase to the superoxide-generating enzyme xanthine oxidase. This may occur during exercise as well as with any tissue ischemia. When exercise reaches an anaerobic threshold, the anaerobic metabolism gives rise to local acidosis (i.e., the Bohr effect). ${ }^{33}$ This acidic environment promotes oxygen release from hemoglobin, increases $\mathrm{PO}_{2}$ in tissues, and releases iron from transferrin. Simultaneously, intrinsic antioxidants are recruited to bring about homeostasis. This results in a decrease or near depletion of antioxidants (such as oxidized glutathione, or vitamins $($ or $E$ ) and stimulation of an antioxidant enzyme (such as superoxide dismutase) as the body's compensatory mechanism. ${ }^{31,34}$

\section{Regular exercise}

The threshold at which oxidative stress occurs in a single bout of exercise seems to depend on the training status of the individual. ${ }^{11}$ The magnitude of adaptation due to regular exercise co- incides largely with the magnitude of prooxidant generation during a single bout of exercise. Exercise at moderate intensity, when repeated, induces several changes at the cellular level that may counteract the increased prooxidant generation more efficiently. ${ }^{30,35}$

Studies with animal models suggest that the intermediary metabolism adapts to the increasing demand of energy generation with a higher concentration of relevant enzymes in mitochondria by increasing the number of mitochondria rather than by increasing the enzymatic content in each mitochondrion. ${ }^{29}$ The beneficial effect of regular exercise, then, may be that each mitochondrion has a relatively lower oxidative load because of the increased number of mitochondria. As a result, each mitochondrion generates less prooxidant. This theory notwithstanding, the effects of training on such enzymatic activities are controversial because of lack of sufficient evidence. In some animal models, a positive association has been found, whereas in others, no changes could be detected. ${ }^{36,37}$

Enzymatic antioxidative systems are also enhanced as a result of regular exercise. ${ }^{38}$ Longterm training is believed to increase the activity of certain antioxidant enzymes in the skeletal muscles and erythrocytes. ${ }^{39}$ Specific antioxidant enzymes work to eliminate reactive oxygen species produced by exercise. Superoxide dismutase, catalase, glutathione peroxidase, and glutathione reductase all participate in eliminating reactive oxygen species and preventing cellular injury. ${ }^{37}$ These antioxidant enzymes are specifically localized in cellular organelles and the cytosol within the cells. ${ }^{38}$ Cross-sectional data from young male volunteers as well as prospective data from middle-aged women have demonstrated a relationship between oxidative capacity and the activities of the free radical scavenging enzymes. Jenkins et al. ${ }^{40}$ and Hernandez at al. ${ }^{30}$ observed a significant correlation between antioxidant enzymes (superoxide dismutase and catalase) and whole body maximal oxygen uptake (i.e., the best index for one's aerobic fitness level). Resistance against oxidative stress preserves the normal $\mathrm{pH}$ level in tissues, thus preventing the production of loosely bound iron. This in turn protects tissue against damage due to prooxidants. ${ }^{39}$ Thus, in humans, training effects have been identified with an enhanced activity of antioxidative enzymes. 


\section{INTERACTION BETWEEN EXERCISE TRAINING AND DEVELOPMENT OF PREECLAMPSIA}

The effects of physical exercise that counteract the development of preeclampsia may be twofold. First, regular exercise may improve some risk factors associated with preeclampsia; for example, obesity is associated with less daily physical activity during pregnancy. ${ }^{41}$ Also, hypertrigyceridemia $^{41}$ and insulin resistance ${ }^{42}$ are reported to improve by regular exercise of moderate intensity during pregnancy. Second, a more direct impact of regular exercise on prevention of preeclampsia may rest in the fact that exercise training enhances resistance to oxidative stress, and exercise enhances expression and activity of superoxide dismutase (Fig. 2). A recent study indicates that women who developed preeclampsia had significantly lower prostacyclin levels throughout pregnancy, even at 13-16 weeks gestation, than women without preeclampsia, but the level of thromboxane $\mathrm{A}_{2}$ did not differ. ${ }^{43}$ The results of this study further endorse the hypothesis that enhanced oxidative stress exists well before the onset of clinical manifestation of preeclampsia.

Some evidence suggests that physically active women are less likely to develop preeclampsia. Marcoux et al. ${ }^{8}$ conducted a retrospective casecontrol study to examine the relation between leisure time physical activity (LTPA) during the first 20 weeks of pregnancy and the risk of de- veloping preeclampsia and gestational hypertension. They report a tendency for women who performed regular LTPA to have a reduced risk of preeclampsia. To date, this study is the only study providing suggestive evidence that those who are physically active tend to have a lower likelihood of developing preeclampsia. Although convincing, the generalizability of the study was limited by its methodology. Only a clinical randomized trial can prove such a hypothesis.

The chronic effects of exercise, as described in the previous section, in theory should counteract the enhancement of oxidative stress, thus interfering with the process that leads to endothelial dysfunction. It seems unlikely that exercise can reverse or even slow down the disease after endothelial dysfunction leads to the final stages, that is, activation of the coagulation cascade, increased vascular reactivity, or loss of vascular integrity. Nevertheless, there are specific mechanisms of adaptation to regular exercise that should reduce risk factors that enhance oxidative stress in women during pregnancy.

One adaptation that regular exercise brings to women's bodies is resistance to production of prooxidants by increasing the number of mitochondria. This resistance against oxidative stress should discourage or slow down the process of lipid peroxidation. Increased iron-binding capacity in serum also should discourage the process of lipid peroxidation, as its propagation requires the presence of a low molecular weight pool of iron or other transition metals. ${ }^{12}$

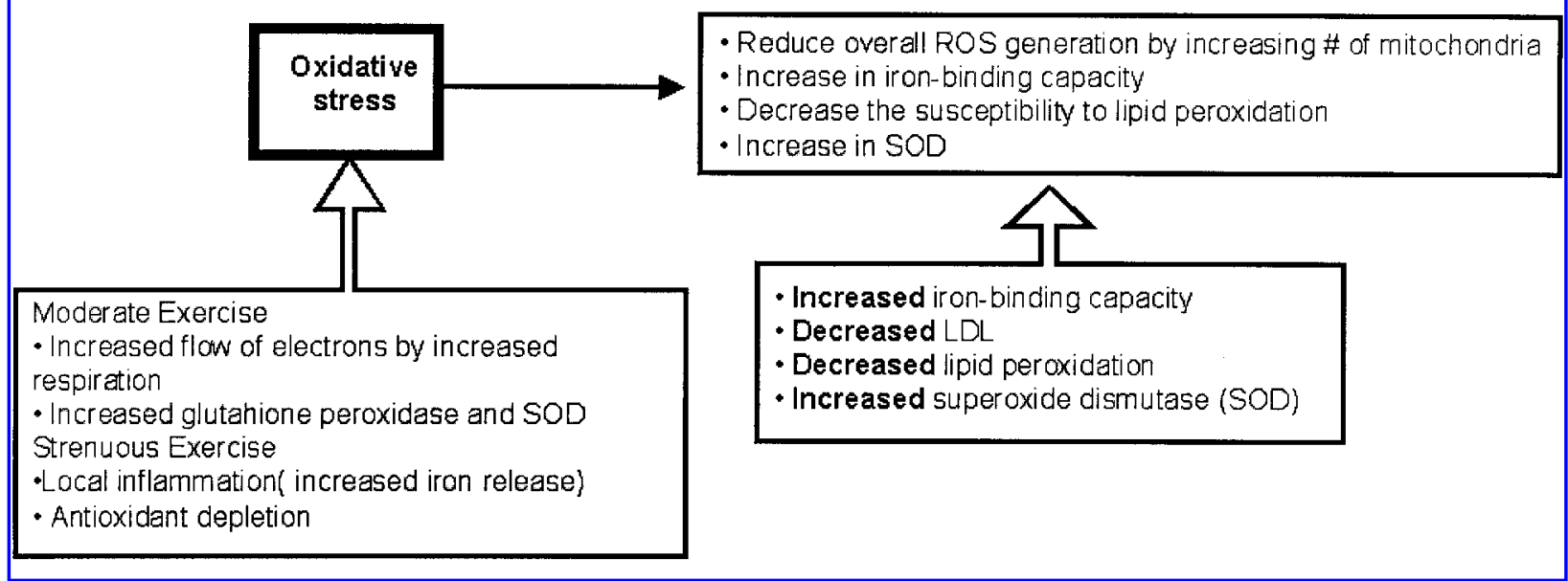

FIG. 2. The role of oxidative stress in acute and regular exercise. 
Equally important is a training effect that decreases susceptibility to lipid peroxidation. Circulating free fatty acids are already increased by 15-20 weeks of gestation in women with preeclampsia. Oxygen free radicals can facilitate the formation of lipid peroxides. Preeclamptic lesions of decidual vessels resemble atherosclerotic lesions, both showing fibrinoid necrosis of the vessel wall and accumulation of lipid-laden form cells, a hallmark of oxidized low-density lipoprotein (LDL). ${ }^{12}$ Decreased antioxidant enzymes, particularly superoxide dismutase, are described as one characteristic of women with preeclampsia. Davidge et al. ${ }^{44}$ report that sera from women with preeclamptic pregnancies had one-half the antioxidant activity of sera from women with uncomplicated pregnancies. Furthermore, increased superoxide dismutase availability, triggered by oxidative stress, is presented as one major adaptation of regular exercise. Physical exercise is reported to induce a significant increase in antioxidant protection via superoxide dismutase, catalase, and glutathione peroxidase activities in blood platelets. ${ }^{45}$ Enhancement of resistance to oxidative stress, one of the proposed effects of exercise training, may reduce the susceptibility toward abnormal pregnancy.

\section{CONCLUSIONS}

Possible mechanisms by which regular exercise might interfere with the development of preeclampsia were discussed in this paper. Although antioxidants can be taken supplementally and proper intake of dietary supplements has a place in healthcare practice, it is important to understand the effect of a large amount of antioxidants on the fetus. Moreover, oxidative stress in preeclampsia is most likely the result of multiple prooxidant pathways, and concerted action of multiple antioxidants should be preferred to afford protection. Finally, unlike previously attempted preventive treatments, such as low-dose aspirin or calcium supplement, that provided limited benefit, regular exercise of moderate intensity leads to a positive and healthy lifestyle without concerns for side effects. The childbearing period often coincides with the beginning of adult life, when the possibility of chronic illness gradually increases. A positive motivation to initiate regular exercise for women may promote a life-long healthy habit of regular exercise. A randomized clinical trial to test the effect of regular exercise on the incidence of preeclampsia is in progress.

\section{REFERENCES}

1. Ansari MZ, Mueller BA, Krohn MA. Epidemiology of eclampsia. Eur J Epidemiol 1995;11:447.

2. Sibai BM, et al. Risk factors associated with preeclampsia in healthy nulliparous women. The Calcium for Preeclampsia Prevention (CPEP) Study Group. Am J Obstet Gynecol 1997;177:1003.

3. Sibai $\mathrm{BM}$, et al. Risk factors for preeclampsia in healthy nulliparous women: A prospective multicenter study. The National Institute of Child Health and Human Development Network of Maternal-Fetal Medicine Units. Am J Obstet Gynecol 1995;172:642.

4. Caritis $\mathrm{S}$, et al. Predictors of pre-eclampsia in women at high risk. National Institute of Child Health and Human Development Network of Maternal-Fetal Medicine Units. Am J Obstet Gynecol 1998;179:946.

5. Dekker GA, Robillard PY, Hulsey TC. Immune maladaptation in the etiology of preeclampsia: A review of corroborative epidemiologic studies. Obstet Gynecol Surv 1998;53:377.

6. Davidge ST. Oxidative stress and altered endothelial cell function in preeclampsia. Semin Reprod Endocrinol 1998;16:65.

7. Ji LL, et al. Oxidative stress and aging. Role of exercise and its influences on antioxidant systems. Ann NY Acad Sci 1998;854:102.

8. Marcoux S, Brisson J, Fabia J. The effect of leisure time physical activity on the risk of pre-eclampsia and gestational hypertension. I Epidemiol Community Health 1989;43:147.

9. Harfenist E, Murray R. Plasma proteins, immunoglobins, \& blood coagulation. In: Harper's biochemistry. East Norwalk, CT: Appleton \& Lange, 1993:665.

10. Esterbauer H, Wag G, Puhl H. Lipid peroxidation and its role in atherosclerosis. Br Med Bull 1993;49:566.

11. Clarkson PM. Antioxidants and physical performance. Crit Rev Food Sci Nutr 1995;35:131.

12. Dekker GA, Sibai GM. Etiology and pathogenesis of preeclampsia: Current concepts. Am J Obstet Gynecol 1998;179:1359.

13. Cargnoni A, et al. Lipid peroxidation in normal pregnancy and preeclampsia. Adv Exp Med Biol 1994;366: 420.

14. Shouk TA, Omar MN, Fayed ST. Essential fatty acids profile and lipid peroxides in severe pre-eclampsia. Ann Clin Biochem 1999;36:62.

15. Walsh SW, Wang Y. Trophoblast and placental villous core production of lipid peroxides, thromboxane, and prostacyclin in preeclampsia. I Clin Endocrinol Metab 1995;80:1888.

16. Hubel CA, et al. Lipid peroxidation in pregnancy: 
New perspectives on preeclampsia. Am J Obstet Gynecol 1989;161:1025.

17. Kloner RA, Przyklenk K, Whittaker P. Deleterious effects of oxygen radicals in ischemia/reperfusion. Resolved and unresolved tissues. Circulation 1989;80: 1115.

18. Lorentzen B, et al. Fatty acid pattern of esterified and free fatty acids in sera of women with normal and pre-eclamptic pregnancy. Br J Obstet Gynaecol 1995; 102:530.

19. Lorentzen B, Henriksen T. Plasma lipids and vascular dysfunction in preeclampsia. Semin Reprod Endocrinol 1998;16:33.

20. Chappell LC, et al. Effects of antioxidants on the occurrence of pre-eclampsia in women at increased risk: A randomised trial. Lancet 1999;354:810.

21. Dekker GA, Sibai BM. The immunology of preeclampsia. Semin Perinatal 1999;23:24.

22. Roggensack AM, Zhang Y, Davidge ST. Evidence for peroxynitrite formation in the vasculature of women with preeclampsia. Hypertension 1999;33:83.

23. Walsh SW. Maternal-placental interactions of oxidative stress and antioxidants in preeclampsia. Semin Reprod Endocrinol 1998;16:93.

24. Hubel CA. Dyslipidemia, iron, and oxidative stress in preeclampsia: Assessment of maternal and feto-placental interactions. Semin Reprod Endocrinol 1998;16: 75.

25. Dekker GA, van Geijn HP. Endothelial dysfunction in preeclampsia. Part I: Primary prevention. Therapeutic perspectives. I Perinat Med 1996;24:99.

26. Katusic ZS, Vanhoutte PM. Superoxide anion and endothelial regulation of arterial tone. Semin Perinatol 1991;15:30.

27. Myatt $\mathrm{L}$, et al. Nitrotyrosine residues in placenta. Evidence of peroxynitrite formation and action. Hypertension 1996;28:488.

28. Hubel CA, et al. Decreased transferrin and increased transferrin saturation in sera of women with preeclampsia: Implications for oxidative stress. $\mathrm{Am} \mathrm{J} \mathrm{Ob-}$ stet Gynecol 1996;175:692.

29. Moller P, Wallin H, Knudsen LE. Oxidative stress associated with exercise, psychological stress and lifestyle factors [Review]. Chem Biol Interact 1996;102:17.

30. Hernandez R, et al. Effects of physical exercise in preand postmenopausal women on lipid peroxidation and antioxidant systems. Endocr Res 1999;25:153.

31. Leaf DA, et al. The effect of exercise intensity on lipid peroxidation. Med Sports Exerc 1997;29:1036.

32. Leaf DA, et al. The exercise-induced oxidative stress paradox: The effects of physical exercise training. Am J Med Sci 1999;317:295.

33. Powers SK, Ji LL, Leeuwenburgh C. Exercise traininginduced alterations in skeletal muscle antioxidant capacity: A brief review. Med Sci Sports Exerc 1999;31: 987.

34. Ji LL. Exercise, oxidative stress, and antioxidants. Am J Sports Med 1996;24(Suppl 6):S20.

35. Kostka $\mathrm{T}$, et al. Physical activity, fitness and integrated antioxidant system in healthy active elderly women. Int J Sports Med 1998;19:462.

36. Higuchi $\mathrm{M}$, et al. Superoxide dismutase and catalase in skeletal muscle: Adaptive response to exercise. I Gerontol 1985;40:281.

37. Laughlin $\mathrm{MH}$, et al. Skeletal muscle oxidative capacity, antioxidant enzymes, and exercise training. J Appl Physiol 1990;68:2337.

38. Fielding RA, Meydani M. Exercise, free radical generation, and aging. Aging (Milano) 1997;9:12.

39. Radak $Z$, et al. Superoxide dismutase derivative reduces oxidative damage in skeletal muscle of rats during exhaustive exercise. J Appl Physiol 1995;79:129.

40. Jenkins RR, Friedland R, Howald $H$. The relationship of oxygen uptake to superoxide dismutase and catalase activity in human skeletal muscle. Int J Sports Med 1984;5:11.

41. Taylor $\mathrm{R}$, et al. Dietary intake, exercise, obesity and noncommunicable disease in rural and urban populations of three Pacific Island countries. I Am Coll Nutr 1992;11:283.

42. Ziogas GG, Thomas TR, Harris WS. Exercise training, postprandial hypertriglyceridemia, and LDL subfraction distribution. Med Sci Sports Exerc 1997;29:986.

43. Mills JL, et al. Prostacyclin and thromboxane changes predating clinical onset of preeclampsia: A multicenter prospective study. JAMA 1999;282:356.

44. Davidge ST, et al. Sera antioxidant activity in uncomplicated and preeclamptic pregnancies. Obstet Gynecol 1992;79:897.

45. Kedziora J, Buczynski A, Kedziora-Kornatowska K. Effect of physical exercise on antioxidative enzymatic defense in blood platelets from healthy men. Int J Occup Med Environ Health 1995;8:33.

Address reprint requests to: SeonAe Yeo, R.N., Ph.D. 400 North Ingalls Ann Arbor, MI 48104 
This article has been cited by:

1. SeonAe Yeo. 2009. Adherence to walking or stretching, and risk of preeclampsia in sedentary pregnant women. Research in Nursing \& Health n/a-n/a. [CrossRef] 